\title{
Bringing new medicines to women with epithelial ovarian cancer: what is the unmet medical need?
}

Thomas J. Herzog ${ }^{1 *}$ and Bradley J. Monk ${ }^{2}$

\begin{abstract}
Background: Therapy for advanced epithelial ovarian cancer (OC) includes first line platinum/taxane-containing chemotherapy and re-treatment with platinum-containing regimens for disease recurrence in patients likely to respond again. Single-agent, non-platinum, cytotoxic agents are commonly used to treat patients resistant to platinum retreatment, but these agents are associated with dose-limiting toxicities and response rates below 20\%.

Main body: Recent advances have led to novel targeted treatments for recurrent $\mathrm{OC}$ that offer opportunities to improve response rates and prolong progression-free intervals. However, they also add complexity to the process of selecting treatment for individual patients at different stages of the disease process. Advanced and recurrent OC is rarely cured. Multiple lines of platinum combinations, and nonplatinum chemotherapeutics eventually fail to achieve clinical benefit, thus other active and tolerable systemic therapies are needed. Consequently, the US Food and Drug Administration has created a mechanism for "accelerated approval" of new medicines in situations of high unmet medical need.
\end{abstract}

Conclusion: We review the clinical implications of recent key clinical studies in these settings and outline the path forward for study design and approval of novel therapeutics to treat recurrent OC.

Keywords: BRCA1/2, Ovarian cancer, PARP, Platinum-refractory

\section{Introduction}

More than $70 \%$ of women with epithelial ovarian cancer (OC), which typically also includes fallopian tube and primary peritoneal cancers, have advanced disease at the time of first diagnosis. Although many patients with advanced disease achieve complete remission after surgical cytoreduction and platinum- and taxane-based chemotherapy, up to $80 \%$ eventually experience recurrence [1]. Two major goals of recent and ongoing clinical studies in OC have been to achieve a more durable disease-free interval after induction therapy and better response rates for regimens administered beyond first line therapy. We provide a succinct overview of recent studies addressing these two goals and outline the unmet need for additional treatment options.

\footnotetext{
* Correspondence: herzogtj@ucmail.uc.edu

${ }^{1}$ University of Cincinnati Cancer Institute, University of Cincinnati, Medical Sciences Bldg, Suite 2005H, ML0662, 231 Albert Sabin Way, Cincinnati, OH 45267-0662, USA

Full list of author information is available at the end of the article
}

\section{Review}

What is the role of maintenance therapy as part of first line therapy?

Platinum-containing induction chemotherapy remains a standard first-line treatment for women with advanced OC. However, there has been vigorous debate regarding the role of maintenance chemotherapy in patients with advanced $\mathrm{OC}$ who achieve an objective response during induction chemotherapy $[1,2]$. In the 1990s, studies of extended platinum chemotherapy (8-12 cycles) found no evidence for improved progression-free survival (PFS) or overall survival (OS) versus 5-6 cycles (reviewed in Markman 2015) [2]. Furthermore, extended platinum regimens were associated with increased toxicity versus standard regimens.

In the early 2000s, a true maintenance study assessed paclitaxel maintenance therapy in women who had achieved an objective complete response to induction platinum-paclitaxel [3]. Patients were randomized to either 12 or 3 additional cycles of single-agent paclitaxel. 
The study was terminated early because patients in the 12-cycle arm had significantly longer PFS than patients in the 3-cycle arm. At mature follow-up, PFS was 22 versus 14 months $(P=0.006)$, but there was no significant effect on OS (53 versus 48 months; $P=0.34$ ) [4]. The lack of a significant effect on OS may have several explanations, including exposure to subsequent active treatment regimens [2]. Patients in the 12-cycle arm experienced higher rates of peripheral neuropathy. Another study of single-agent paclitaxel $(6$ cycles) after complete or pathologic response to platinum-paclitaxel induction demonstrated no improvement in PFS or OS, and increased rates of peripheral neuropathy in the paclitaxel arm [5]. Recently, those findings were confirmed by another phase 3 study (GOG-212), which also demonstrated no OS benefit for patients who received maintenance paclitaxel [6].

Two studies (GOG-218 and ICON7) have explored use of the anti-angiogenesis agent bevacizumab to extend the disease-free interval after first line chemotherapy $[7,8]$. In both studies, bevacizumab was added to standard chemotherapy (5 or 6 cycles of carboplatinpaclitaxel), and bevacizumab monotherapy was continued (for 12-22 cycles) after cessation of chemotherapy. Initially, both studies reported improved PFS when extended bevacizumab treatment was added to chemotherapy $[7,8]$. However, long-term follow-up of the ICON7 study found no significant improvement in PFS or OS in the overall study population, although there was evidence of benefit in high-risk patients [9]. The GOG-218 study had three treatment arms, all of which received 6 cycles of carboplatin-paclitaxel [7]. One arm received bevacizumab concurrent with chemotherapy, a second arm received bevacizumab concurrently and during an extended period (up to cycle 22), and the third arm received only chemotherapy. A placebo was administered as appropriate control in this double-blind study. $\mathrm{Pa}-$ tients in the extended-bevacizumab arm had the longest PFS (14.1 months), which was significantly longer than the chemotherapy-alone arm (10.3 months; hazard ratio [HR]: 0.717; 95\% confidence interval [CI]: 0.625-0.824; $P<0.001)$. The PFS in the concurrent-bevacizumab arm was 11.2 months [7]. In both studies, bevacizumab was associated with increased risk of adverse events, especially gastrointestinal events [10], and neither study found a benefit in terms of OS for the overall study population. Exposure to bevacizumab or other active regimens after the study may have confounded any OS effect; nonetheless, the role of bevacizumab in front-line therapy for advanced OC-either concurrently with chemotherapy or for an extended duration-continues to be controversial. SOLO-1 is an ongoing study of olaparib maintenance monotherapy after first line platinum-based chemotherapy. Also, the randomized, phase 3 PAOLA-1 study (NCT02477644) is comparing olaparib and bevacizumab versus placebo and bevacizumab as maintenance therapy in patients with advanced OC following first line treatment with platinum chemotherapy and bevacizumab [11]. Olaparib and other Poly (ADP-ribose) polymerase (PARP) inhibitors are discussed in more detail below.

\section{Recurrent ovarian cancer}

Selection of treatment for recurrence of advanced epithelial $\mathrm{OC}$ is generally guided by the progression-free interval [12] (Fig. 1). When the time to progression is $>6$ months after cessation of initial platinum-containing chemotherapy, the disease is considered to be platinumsensitive. In these patients, treatment using a combination platinum-containing chemotherapy (typically including a taxane, pegylated liposomal doxorubicin [PLD], gemcitabine, or bevacizumab in carefully selected patients) is considered a preferred treatment option [13]. Response rates to second line, combination platinumcontaining chemotherapy in patients with platinumsensitive tumors are approximately 50\%-65\% [14-16]. Combination therapy demonstrated advantages over single-agent platinum regimens in terms of both PFS and OS [17]. When progression after first line therapy occurs less than 6 months after cessation of chemotherapy, the disease is considered to be platinum-resistant; recommended second line therapies in these patients include mostly single-agent, nonplatinum-containing chemotherapy regimens, with the possible addition of bevacizumab or pazopanib in carefully selected patients [13]. When progression occurs during chemotherapy or within 1 month of cessation, the disease is considered platinum-refractory [12].

Until recent years, there were essentially no treatment options other than repeated courses of chemotherapy in patients with 2 or more prior lines of chemotherapy. Furthermore, nearly all patients eventually become resistant to platinum-containing regimens [12]. Thus, the concept of platinum sensitivity becomes less important beyond 2 or 3 lines of chemotherapy, and its relevance to treatment selection in such patients is not well understood. The limited available evidence indicates that responsiveness to platinum-containing regimens declines dramatically after 2 prior lines, even in patients who were initially platinum-sensitive. In one study of 63 patients who received at least 3 lines of chemotherapy, only $11.9 \%$ had a clinical response to third line chemotherapy, although $52 \%$ had responded to second line [18]. Nonplatinum-containing regimens-such as PLD, paclitaxel, gemcitabine, or topotecan-have similar response profiles (range 10\%-15\%), PFS (3-4 months), and OS ( 12 months) when used as late-line therapies [12]. A retrospective analysis of 3 large European clinical 


Platinum-based
chemotherapy

studies of chemotherapy in patients with OC $(N=1620)$ found that the benefits of chemotherapy in terms of increased PFS or OS declined with successive recurrences (Fig. 2) [19]. Although chemotherapy for a fourth recurrence was still associated with a small benefit in terms of OS, the authors concluded that this benefit was mostly due to patients with platinum-sensitive disease, and that chemotherapy beyond three recurrences was not beneficial in patients with platinum-resistant disease. This conclusion, however, may need to be revisited as more data accumulate from studies of patient subgroups, and from new and emerging treatment strategies for patients with multiple recurrences.

Because of reduced potential for benefit after 2 prior lines of chemotherapy, and severe effects on quality of life (QOL), some patients forego chemotherapy after 2 prior lines, although many patients prefer to continue receiving additional lines of chemotherapy even if it confers diminishing benefits [20]. Undoubtedly, many patients withstand the side effects from subsequent lines of chemotherapy but obtain limited benefit. Furthermore, repeated chemotherapy regimens may expose patients to cumulative toxicities associated with many of these regimens $[1,19]$.

Recently, molecularly targeted inhibitors of vascular endothelial growth factor (VEGF; ie, bevacizumab) and PARP (ie, olaparib, rucaparib, and niraparib) have emerged as treatment options in patients with advanced epithelial OC after multiple prior lines of chemotherapy (Table 1) [21-32]. Both bevacizumab and PARP inhibitors require careful patient selection, the criteria for which are still evolving.

\section{Which patients are candidates for bevacizumab in recurrent $\mathrm{OC}$}

Bevacizumab, in combination with paclitaxel, PLD, or topotecan is approved for the treatment of patients with platinum-resistant recurrent epithelial $\mathrm{OC}$ who received no more than 2 prior lines of chemotherapy [21, 22, 25, 33]. In the AURELIA study, patients with platinum-resistant OC and 2 or fewer prior lines of chemotherapy had response rates of $27.3 \%$ in the bevacizumab plus chemotherapy arm versus $11.8 \%$ in the chemotherapyalone $\operatorname{arm}(P=0.001)$. Note that this study excluded patients who were platinum-refractory (progression during previous platinum-containing therapy) [25].

In patients with platinum-sensitive, recurrent $\mathrm{OC}$, the OCEANS study found significantly increased objective response rate (ORR; 78\% versus 57\%; $P<0.001$ ) and PFS (12.4 versus 8.4 months; $P<0.001$ ) when bevacizumab was added to gemcitabine plus carboplatin, but there was no improvement in OS [26, 27]. Another randomized phase 3 study (GOG-213) found a statistically significant improvement in response rate $(78 \%$ versus $59 \%$ in a subset of patients with evaluable data from imaging; $P<0.001)$ and PFS (13.8 versus 10.4 months; $P<0.001)$ when bevacizumab was added to paclitaxel plus carboplatin [28]. In the primary analysis of this study, OS was better in the group that received bevacizumab (42.2 versus 37.7 months), although this incremental improvement narrowly missed achieving statistical significance $(P=0.056)$. However, when miscalculations of the prior platinum-free interval were corrected, the difference in OS achieved statistical significance $(P=0.045)$ [28].

Single-agent bevacizumab has also shown activity (clinical response rate $16 \%-21 \%$ ) in patients with 1 to 3 prior lines of chemotherapy, most of whom were platinum-resistant $[21,22]$. Other small studies have reported responses to single-agent bevacizumab as a later line of therapy in patients with recurrent, platinumresistant $\mathrm{OC}$, with response rates ranging from $13 \%$ to $16 \%[21,23,24]$.

Thus, there is evidence to support the use of bevacizumab in multiple settings, including in combination with chemotherapy (typically PLD, weekly paclitaxel, or topotecan) in platinum-resistant patients with no more than 2 

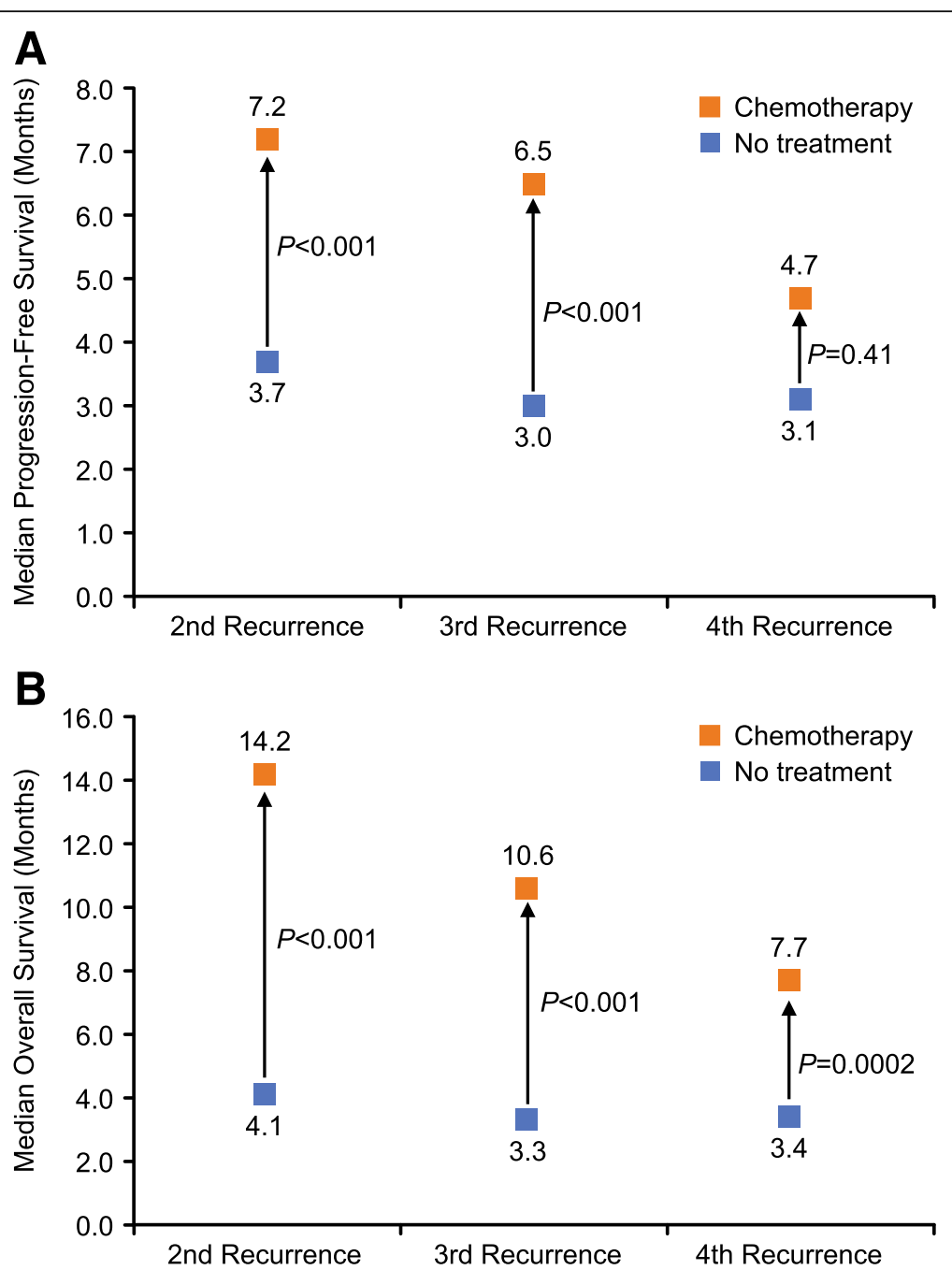

Fig. 2 Median Progression-Free Survival (a) and Overall Survival (b) Associated with Successive Lines of Chemotherapy (Versus no Treatment) in a Retrospective Analysis of Three Randomized Trials in Patients with Advanced Ovarian Cancer [19]. Data from Hanker et al. Ann Oncol. 2012;23:2605-12. Hanker et al. performed a retrospective, pooled analysis of three randomized, phase 3 studies of primary taxane-platinum-based chemotherapy. The analysis included 1620 patients for whom complete data were available. Responsiveness to platinum-containing regimens declined dramatically after 2 prior lines, even in patients who were initially platinum-sensitive [19]

prior lines of chemotherapy. But bevacizumab may also provide benefit in platinum-sensitive patients in combination with carboplatin-gemcitabine or carboplatinpaclitaxel. The United States Food and Drug Administration (US FDA)-approved indications for bevacizumab in OC are summarized in Table 2 [33]. Perhaps just as important to the selection of patients for bevacizumab is the strict exclusion of patients who are at increased risk of bowel perforation. Restrictive exclusion criteria used in clinical studies are commonly followed $[21,25]$. These criteria exclude any patient with a history of bowel obstruction (including subocclusive disease) related to underlying disease, history of abdominal fistula, gastrointestinal perforation, intra-abdominal abscess, evidence of rectosigmoidal involvement by pelvic exam, bowel involvement on computed tomography, or clinical symptoms of bowel obstruction [25]. The role of bevacizumab after previous exposure requires further study; thus, some clinicians will withhold repeat courses until further data are reported.

\section{What is the role of PARP inhibitors for treatment of recurrent $\mathrm{OC}$ ?}

Recent clinical studies have evaluated the potential roles of PARP inhibitors for treatment of patients with advanced $O C$ in two distinct settings: 1) when disease has recurred or progressed after $2-3$ or more prior lines of platinum-containing chemotherapy, and 2) when the disease is in a state of response after completion of a recent course of platinum-containing chemotherapy (maintenance therapy). Some of these studies enrolled only patients with known deleterious $B R C A$ mutations (germline or somatic). 
Table 1 Clinical activity of targeted therapies for treatment of recurrent ovarian cancer in heavily pretreated patients

\begin{tabular}{|c|c|c|c|c|c|c|c|c|}
\hline & Phase & Patients with $\mathrm{OC}, \mathrm{n}$ & Previous therapies & ORR, n (\%) & $C R, n(\%)$ & Median DoR, mo & Median PFS, mo & OS, mo \\
\hline \multicolumn{9}{|c|}{ Bevacizumab Monotherapy } \\
\hline Cannistra 2007 [21] & 2 & 44 & $2-3$ & $7(16)$ & 0 & 4.2 & 4.4 & 10.7 \\
\hline Burger 2007 [22] & 2 & 62 & $1-2$ & $13(21)$ & $2(3)$ & 10.3 & 4.7 & 16.9 \\
\hline Monk 2006 [23] & & 32 & 5 (range: $2-10$ ) & $5(16)$ & $1(3)$ & $N R$ & 5.5 & 6.9 \\
\hline Pietzner 2011 [24] & & 15 & 5.4 (range: $1-7$ ) & $2(13)$ & 0 & $N R$ & NR & 15.0 \\
\hline \multicolumn{9}{|c|}{ Bevacizumab-Chemotherapy Combination } \\
\hline AURELIA [25] & 3 & 361 & $\leq 2$ & $27.3 \%$ & NR & $N R$ & 6.7 & 16.6 \\
\hline OCEANS $[26,27]$ & 2 & 484 & $\leq 1$ & 190/242 (78.5) & $42(17)$ & 10.4 & 12.4 & 33.6 \\
\hline GOG-213 [28] & 3 & 674 & $\geq 3$ & 196/249 (78) & $79 / 249(32)$ & NR & 13.8 & 42.2 \\
\hline \multicolumn{9}{|l|}{ Olaparib Monotherapy } \\
\hline Kaufman 2015 [29] & 2 & 193 & $4.3 \pm 2.2(\mathrm{SD})$ & $60(31)$ & $6(3)$ & 7.5 & 7 & 16.6 \\
\hline$\geq 3$ Prior lines $[30]^{a}$ & & $137^{\mathrm{a}}$ & $\geq 3$ & $46(34)$ & $2(2)$ & 7.9 & 6.7 & NR \\
\hline Gelmon 2011 [31] & 2 & 65 & 3 (range: 1-10) & $18(29)$ & 0 & NR & 7.3 & NR \\
\hline \multicolumn{9}{|l|}{ Rucaparib Monotherapy } \\
\hline Swisher 2017 [32] & 2 & & & & & & & \\
\hline$B R C A$ mutant & & 40 & $1-2$ & $32(80)$ & NR & 9.2 & 12.8 & NR \\
\hline \multicolumn{9}{|l|}{$B R C A$ wild-type } \\
\hline LOH high & & 82 & $1-2$ & $24(29)$ & NR & 10.8 & 5.7 & NR \\
\hline LOH low & & 70 & $1-2$ & $7(10)$ & & 5.6 & 5.2 & \\
\hline
\end{tabular}

$C R$ complete response, $D o R$ duration of response, $L O H$ loss-of-heterozygosity score, mo months, $N R$ not reported, $O C$ ovarian cancer, ORR objective/overall response rate OS overall survival, PFS progression-free survival, SD standard deviation

${ }^{a}$ Subset of patients in Kaufman/Domchek who had measurable disease at baseline and $\geq 3$ prior lines of chemotherapy

\section{Which patients with recurrent ovarian cancer are candidates for a PARP inhibitor?}

In late 2014, olaparib received accelerated approval by the US FDA as monotherapy for patients with advanced OC harboring deleterious or suspected deleterious germline $B R C A$ (gBRCA) mutations and who were previously treated with 3 or more lines of chemotherapy. Approval was primarily based on data from a single-arm study of patients with advanced $\mathrm{OC}$ and gBRCA1/2 mutations $[29,30]$, most of whom had received multiple prior lines of chemotherapy (mean 4.3). Among 137 patients who had measurable disease at baseline and who had received 3 or more prior lines of chemotherapy, the ORR was $34 \%$ and the median duration of response was 7.9 months (Table 1) [30]. Although patients with platinum-sensitive disease had the highest ORR (18/39; $46 \%)$, the response rate observed in patients with platinum-resistant disease $(24 / 81 ; 30 \%)$ suggests that platinum resistance does not preclude responsiveness to olaparib as late-line therapy in patients with $\mathrm{OC}$ and a $B R C A$ mutation. In contrast, the ORR was $14 \%(2 / 14)$ among patients with platinum-refractory disease [30].

In late 2016, another PARP inhibitor, rucaparib (Table 2), was approved for treatment of patients with advanced OC associated with deleterious BRCA mutation (germline or somatic) and who had progressed after 2 or more prior lines of chemotherapy. The accelerated approval was based upon ORR (54\%) [34] and median duration of response (9.2 months) in patients with a $B R C A$ mutation from the single-arm, phase 2 study (ARIEL2 Part 1) that enrolled women with high-grade, relapsed, platinum-sensitive OC [32]. The ARIEL2 study results are shown in Table 1 [32].

One of the goals of the ARIEL2 study was to explore biomarkers of response to PARP inhibition. Thus, the study also included patients who had wild-type $B R C A$, but tumor samples were analyzed for genetic loss of heterozygosity $(\mathrm{LOH})$ as a potential surrogate marker of homologous recombination deficiency (HRD). Although patients with wild-type $B R C A$ but high $\mathrm{LOH}$ scores had lower rates of ORR and shorter PFS than patients with $B R C A$ mutation, both cohorts (those with $B R C A$ mutation and those with wild-type $B R C A$ but high $\mathrm{LOH}$ scores, indicating presence of HRD) had ORR and PFS that were significantly better than patients with wild-type $B R C A$ and low LOH scores $(P<0.02)$ [32]. These results demonstrated that PARP inhibitors appear active in a broader set of patients than only those harboring deleterious BRCA mutations. Part 2 of the ARIEL2 trial is ongoing, and it will prospectively evaluate rucaparib responsiveness in patient subgroups defined by LOH scores. 
Table 2 US FDA-approved targeted therapies for ovarian cancer

\begin{tabular}{|c|c|c|c|c|}
\hline & Drug class & Ovarian cancer indication & Black box warnings & Warnings and precautions \\
\hline $\begin{array}{l}\text { Bevacizumab } \\
\text { [33] }\end{array}$ & $\begin{array}{l}\text { VEGF inhibitor; } \\
\text { anti-angiogenesis }\end{array}$ & $\begin{array}{l}\text { Platinum-resistant recurrent disease } \\
\text { - In combination with paclitaxel, PLD, } \\
\text { or topotecan with no more than } 2 \\
\text { prior lines of chemotherapy } \\
\text { Platinum-sensitive recurrent disease } \\
\text { - In combination with carboplatin and } \\
\text { paclitaxel, or carboplatin and gemcitabine; } \\
\text { followed by single-agent bevacizumab }\end{array}$ & $\begin{array}{l}\text { - Gastrointestinal perforations } \\
\text { - Surgery and wound healing } \\
\text { complications } \\
\text { - Hemorrhage }\end{array}$ & $\begin{array}{l}\text { - Perforation or fistula } \\
\text { - Arterial and venous } \\
\text { thromboembolic events } \\
\text { - Hypertension } \\
\text { - Posterior reversible encephalopathy } \\
\text { syndrome } \\
\text { - Proteinuria } \\
\text { - Infusion reactions } \\
\text { - Embryo-fetal toxicity } \\
\text { - Ovarian failure }\end{array}$ \\
\hline Niraparib [36] & PARP inhibitor & $\begin{array}{l}\text { Maintenance treatment of recurrent } \\
\text { disease in complete or partial response } \\
\text { to platinum-based chemotherapy }\end{array}$ & None & $\begin{array}{l}\text { - Myelodysplastic syndrome/acute } \\
\text { myeloid leukemia } \\
\text { - Bone marrow suppression } \\
\text { - Cardiovascular effects (blood } \\
\text { pressure and heart rate) } \\
\text { - Embryo-fetal toxicity }\end{array}$ \\
\hline Olaparib [35] & PARP inhibitor & $\begin{array}{l}\text { Maintenance treatment of recurrent } \\
\text { disease in complete or partial response } \\
\text { to platinum-based chemotherapy } \\
\text { Treatment of deleterious or suspected } \\
\text { deleterious germline BRCA-mutated } \\
\text { disease with } \geq 3 \text { prior lines of } \\
\text { chemotherapy; requires FDA-approved } \\
\text { companion diagnostic test }\end{array}$ & None & $\begin{array}{l}\text { - Myelodysplastic syndrome/acute } \\
\text { myeloid leukemia } \\
\text { - Pneumonitis } \\
\text { - Embryo-fetal toxicity }\end{array}$ \\
\hline Rucaparib [34] & PARP inhibitor & $\begin{array}{l}\text { Monotherapy in patients with deleterious } \\
\text { BRCA mutations treated with two or more } \\
\text { prior chemotherapies; requires companion } \\
\text { diagnostic test }\end{array}$ & None & $\begin{array}{l}\text { - Myelodysplastic syndrome/acute } \\
\text { myeloid leukemia } \\
\text { - Embryo-fetal toxicity }\end{array}$ \\
\hline
\end{tabular}

PARP poly (ADP-ribose) polymerase, PLD pegylated liposomal doxorubicin, VEGF vascular endothelial growth factor

PARP inhibitors are also associated with side effects, although not usually as severe as those observed with chemotherapy. However, a small percentage (1\% or less) in both olaparib [35] and rucaparib [34] studies developed myelodysplastic syndrome/acute myeloid leukemia. Patients should be monitored for hematologic toxicities at baseline and during treatment.

Is there a role for PARP inhibitors in maintenance therapy? Both olaparib [35] and niraparib [36] (Table 2), have been approved as maintenance therapies in patients who are in a complete or partial response to platinum-based chemotherapy [37-39]. Some have questioned use of the term "maintenance therapy" in this setting, on the basis that many patients had only a partial response to chemotherapy rather than a complete response. Nevertheless, this term has been adopted by regulatory agencies, and it will continue to be used in this context.

The maintenance therapy studies of PARP inhibitors enrolled patients with $\mathrm{OC}$ who were in response (partial or complete) after their most recent platinumcontaining regimen and who had responded for at least 6 months after the preceding platinum-containing regimen. In the phase 2 olaparib study (Study 19), patients in the olaparib arm had significantly longer PFS than those in the placebo arm (8.4 versus 4.8 months; $P<0.001)$ [37]. Further analysis according to $B R C A$ status revealed that patients with a deleterious $B R C A$ mutation (germline or somatic) had the greatest benefit from olaparib versus placebo (PFS 11.2 versus 4.3 months; $P<0.001$ ), but even patients with wild-type $B R C A$ benefited from olaparib maintenance therapy (PFS 7.4 versus 5.5 months; $P=0.007$ ) [40]. While no significant difference in OS was found for the general population, patients with $B R C A$ mutation-positive, platinum-sensitive, recurrent $\mathrm{OC}$ benefited from longer survival when treated with olaparib [41]. The recently reported SOLO-2 study was a confirmatory phase 3 trial to determine the efficacy of olaparib tablets as maintenance monotherapy in patients with platinum-sensitive, relapsed $\mathrm{OC}$ and germline $B R C A$-mutation [39]. In this study, olaparib maintenance therapy was associated with marked improvements in PFS versus placebo (Table 3). Health-related QOL also was evaluated in the SOLO-2 study. Patients maintained their QOL while on olaparib maintenance therapy, exhibiting no significant negative effect on health-related QOL versus placebo. Patients receiving olaparib (versus placebo) experienced a significant improvement in multiple assessments of patientcentered benefits [42].

The phase 3 niraparib study (NOVA) enrolled 2 cohorts of patients according to the presence or absence of a gBRCA mutation [38]. In the gBRCA cohort, niraparib treatment was also associated with markedly longer PFS 
Table 3 Phase 3 studies of PARP inhibitors for maintenance therapy in patients with platinum-sensitive ovarian cancer

\begin{tabular}{|c|c|c|c|c|c|c|}
\hline & \multirow{2}{*}{$\begin{array}{l}\text { Prior lines of } \\
\text { chemotherapy }\end{array}$} & \multirow[t]{2}{*}{ Inclusion biomarkers } & \multicolumn{2}{|l|}{ Median PFS, months } & \multirow{2}{*}{$\begin{array}{l}\text { HR for PFS } \\
(95 \% \mathrm{Cl})\end{array}$} & \multirow[t]{2}{*}{$P$ value } \\
\hline & & & Active therapy & Placebo & & \\
\hline \multicolumn{7}{|c|}{ Niraparib Monotherapy } \\
\hline \multirow[t]{4}{*}{ NOVA [38] } & \multirow[t]{4}{*}{$\geq 2$} & \multirow{4}{*}{$\begin{array}{l}\text { None } \\
\text { Patients stratified according to } \\
\text { gBRCA status and HRD score }\end{array}$} & gBRCA: 21.0 & 5.5 & $0.27(0.17-0.41)$ & $<0.001$ \\
\hline & & & Non-gBRCA: 9.3 & 3.9 & $0.45(0.34-0.61)$ & $<0.001$ \\
\hline & & & HRD-positive: 12.9 & 3.8 & $0.38(0.24-0.59)$ & $<0.001$ \\
\hline & & & HRD-negative: 6.9 & 3.8 & $0.58(0.36-0.92)$ & 0.02 \\
\hline \multicolumn{7}{|c|}{ Olaparib Monotherapy } \\
\hline SOLO-2 [39] & $\geq 2$ & BRCA1/2 mutation & 30.2 & 5.5 & $0.25(0.18-0.35)$ & $<0.001$ \\
\hline \multicolumn{7}{|c|}{ Rucaparib Monotherapy } \\
\hline ARIEL3 $[45,46]$ & $\geq 3^{a}$ & None & $\begin{array}{l}\text { BRCA mutation: } 16.6 \\
\text { HRD-positive: } 13.6 \\
\text { ITT population: } 10.8\end{array}$ & $\begin{array}{l}5.4 \\
5.4 \\
5.4\end{array}$ & $\begin{array}{l}0.23 \\
0.32 \\
0.36\end{array}$ & $\begin{array}{l}<0.001 \\
<0.001 \\
<0.001\end{array}$ \\
\hline
\end{tabular}

$C l$ confidence interval, $g B R C A$ germline BRCA mutation, $H R$ hazard ratio, HRD homologous recombination deficiency, ITT intent-to treat, PARP poly (ADP-ribose) polymerase, PFS progression-free survival

${ }^{a}$ Received $\geq 2$ prior platinum-based treatment regimens including platinum based regimen and no more than 1 non-platinum chemotherapy regimen

than placebo (Table 3). Patients in the non-gBRCA cohort also had significant benefits from niraparib therapy. Interestingly, even patients who did not have a gBRCA mutation, and who did not exhibit HRD, experienced a longer PFS with niraparib versus placebo [38]. In a subgroup analysis, niraparib provided significant benefit in patients with recurrent $\mathrm{OC}$ who achieved a partial response following platinum therapy [43]. In addition, $49 \%$ of the total patient population was found to have developed platinum resistance to previous chemotherapy, yet the study met its primary endpoint of prolonged PFS following response to most recent platinum therapy [44]. Results for OS have not yet been reported from either the SOLO-2 or NOVA studies.

With appropriate caution regarding toxicities, PARP inhibitors are emerging as a potential maintenance therapy in patients with $\mathrm{OC}$ who have responded to at least 2 prior lines of platinum-containing chemotherapy and are in response (complete or partial) to the most recent course. Although BRCA mutations and deficiency in homologous recombination repair appear to be relative markers predictive of response to PARP inhibitors, lack of these markers does not preclude a response. This concept is especially notable in the NOVA trial, which led to recent approval of niraparib irrespective of biomarker status. Thus, neither BRCA status nor HRD score are requisites for use of niraparib. The ongoing ARIEL3 study (NCT01968213) is exploring the use of rucaparib as maintenance therapy, with enrollment criteria similar to those in the olaparib and niraparib maintenance therapy studies, but without restrictions related to BRCA status. As reported in June 2017, the phase 3 ARIEL3 study demonstrated improved PFS (Table 3) by investigator review for rucaparib compared with placebo in all three primary efficacy analyses: $B R C A$ mutation
(16.6 months vs 5.4 months; HR: $0.23, P<0.001$ ); HRDpositive (13.6 months vs 5.4 months; HR: $0.32, P<0.001$ ); overall intent-to-treat populations (10.8 months vs 5.4 months; HR: $0.36, P<0.001)[45,46]$.

\section{What percentage of patients with advanced ovarian cancer have BRCA mutations?}

In population-based studies of unselected patients with OC, $5 \%-18 \%$ of cases were found to be associated with gBRCA mutations [47]. Limited available data suggest that another $5 \%-10 \%$ arise from somatic $B R C A$ mutations [48]. Thus, at initial diagnosis the percentage of patients with $\mathrm{OC}$ whose cancer is $B R C A$-related is modest. However, patients with $B R C A$-related $\mathrm{OC}$ have better long-term survival than non-carriers $[49,50]$, which may in part be related to better responsiveness to platinumbased chemotherapy $[50,51]$. Thus, patient groups who have undergone multiple lines of chemotherapy may become enriched for $B R C A$ mutation carriers.

\section{What are the prospects for the unmet needs of patients requiring third line therapy and beyond?}

Several studies are investigating treatment options for patients who have platinum-resistant disease or who have progressed after multiple lines of treatment, including the third line setting and beyond (Table 4). With regard to PARP inhibitors, the phase 3 SOLO-3 study is measuring PFS for olaparib versus single-agent investigator's choice nonplatinum-based chemotherapy in patients with platinum-sensitive high-grade serous $\mathrm{OC}$ or high-grade endometrioid cancer who progressed at least 6 months after last platinum treatment, and have received 2 or more platinum-based lines of therapy (NCT02282020) [52]. To determine the most sensitive and specific assays to assess HRD and more accurately 
Table 4 Ongoing phase 2 and 3 studies investigating late-line therapies in ovarian cancer

\begin{tabular}{|c|c|c|c|c|c|}
\hline Agent & $\begin{array}{l}\text { NCT \# } \\
\text { Study name }\end{array}$ & Phase & Est. N & Setting & Expected completion \\
\hline \multicolumn{6}{|c|}{ PARP Inhibitors } \\
\hline Niraparib & $\begin{array}{l}\text { NCT02354586 } \\
\text { QUADRA }\end{array}$ & 2 & 400 & Recurrent, $\geq 4$ th- 5 th-line & October 2017 \\
\hline Olaparib & $\begin{array}{l}\text { NCT02282020 } \\
\text { SOLO-3 }\end{array}$ & 3 & 411 & Recurrent, $\geq 3$ rd-line & December 2017 \\
\hline Olaparib & $\begin{array}{l}\text { NCT02889900 } \\
\text { CONCERTO }\end{array}$ & 2 & 100 & Recurrent, $\geq 3$ rd-line & November 2018 \\
\hline Rucaparib & $\begin{array}{l}\text { NCT01891344 } \\
\text { ARIEL2 }\end{array}$ & 2 & 480 & Recurrent, $\geq 4$ th-line & March 2017 \\
\hline Rucaparib & $\begin{array}{l}\text { NCT01968213 } \\
\text { ARIEL3 }\end{array}$ & 3 & 540 & Recurrent, $\geq 3$ rd-line & March 2017 \\
\hline \multicolumn{6}{|c|}{ Mirvetuximab soravtansine (an antibody-drug conjugate targeting the folate-alpha receptor) } \\
\hline & $\begin{array}{l}\text { NCT02631876 } \\
\text { FORWARDI }\end{array}$ & 3 & 333 & $\begin{array}{l}\text { Platinum-resistant; } 1-3 \text { prior lines } \\
\text { of chemotherapy }\end{array}$ & February 2019 \\
\hline \multicolumn{6}{|c|}{ NUC-1031 (gemcitabine prodrug ${ }^{\mathrm{a}}$ ) } \\
\hline & NCT03146663 & 2 & 64 & $\begin{array}{l}\text { Platinum-resistant; } \geq 3 \text { prior lines } \\
\text { of chemotherapy }\end{array}$ & June 2020 \\
\hline \multicolumn{6}{|c|}{ Trabectedin (novel alkylating chemotherapy agent) } \\
\hline & $\begin{array}{l}\text { NCT01846611 } \\
\text { ORCHYD }\end{array}$ & 3 & 670 & $\begin{array}{l}\text { Platinum-sensitive; 3rd line; known } \\
\text { BRCA1/2 mutation }\end{array}$ & December 2019 \\
\hline \multicolumn{6}{|c|}{ Ipilimumab (immune checkpoint inhibitor) } \\
\hline & NCT01611558 & 2 & 49 & $\begin{array}{l}\text { Platinum-sensitive; } \leq 4 \text { prior lines } \\
\text { of chemotherapy }\end{array}$ & July 2019 \\
\hline \multicolumn{6}{|c|}{ Birinapant (SMAC mimetic and IAP inhibitor) } \\
\hline & NCT02756130 & 2 & 34 & $\begin{array}{l}\text { In combination with carboplatin in } \\
\text { newly diagnosed or recurrent disease }\end{array}$ & June 2020 \\
\hline \multicolumn{6}{|c|}{ Volasertib (Plk1 inhibitor) } \\
\hline & & & & $\begin{array}{l}\text { In development; no ongoing phase } \\
2 \text { or phase } 3 \text { studies }\end{array}$ & \\
\hline
\end{tabular}

IAP inhibitor of apoptosis protein, Plk1 polo-like kinase 1, SMAC second mitochondrial-derived activator of caspases

aprodrug is a compound that is metabolized into a pharmacologically active drug after administration

predict patients who may respond to treatment with olaparib, the phase 2 LIGHT study (NCT02983799) is evaluating the efficacy and safety of olaparib in patients with platinum-sensitive, relapsed $\mathrm{OC}$ who have received $\geq 2$ prior lines of platinum-based chemotherapy. Patients will be stratified by use of different HRD genetic tests [53].

The combination of olaparib with cediranib, an antiangiogenic VEGF receptor inhibitor, is of interest. The phase 2 NCI-2012-02938 study (NCT01116648) in women with recurrent platinum-sensitive OC reported significantly longer median PFS for those treated with combination therapy versus olaparib alone (16.5 vs 8.2 months, HR: $0.50, P=0.007)$. This effect was greatest for patients without known gBRCA mutations: median PFS was 23.7 versus 5.7 months (HR: $0.32, P=0.002)$ and median OS was 37.8 versus 23.0 months (HR: 0.48, $P=0.074$ ) with combination therapy versus olaparib alone, respectively. These results suggest that the combination of a PARP inhibitor and an antiangiogenic may result in increased activity in these patients [54]. Ongoing studies for this combination include: the phase 3 NRGGY004 study (NCT02446600) in platinum-sensitive OC compared to olaparib alone or platinum-based chemotherapy [55]; the single arm phase 2 CONCERTO study (NCT02889900) in women with platinum-resistant relapsed disease without a gBRCA mutation [56]; and the phase 2/3 NRG-GY005 study (NCT02502266) in patients with platinum-resistant disease who have received no more than 3 prior treatment regimens [57].

The phase 2 QUADRA study (NCT02354586) is evaluating the antitumor activity of niraparib in patients with advanced, relapsed, high-grade serous epithelial ovarian, fallopian tube, or primary peritoneal cancer who received 3 or more prior chemotherapy regimens [58]. In platinumresistant disease, ongoing studies are investigating the use of the antibody-drug conjugate mirvetuximab soravtansine and the gemcitabine prodrug NUC-103. Other agents are also being investigated in phase 2 studies, including the 
alkylating agent trabectedin, an antibody-drug conjugate targeting the folic acid receptor (mirvetuximab soravtensine), the immune checkpoint inhibitors ipilimumab, durvalumab, and tremelimumab, and the targeted agents birinapant and volasertib (Table 4). AZD1775, an inhibitor of the WEE1 tyrosine kinase, is also is being explored in platinum-resistant OC (NCT02272790) [59]. Numerous other agents are being studied as earlier lines of therapy for advanced OC, including the folic acid receptor antibody farletuzumab and several inhibitors of histone deactylase (HDAC). The HDAC inhibitors most clinically advanced for treatment of OC are entinostat (NCT02915523), vorinostat (NCT00132067), and ricolinostat (NCT02661815). Finally, several vaccines are being studied as potential treatments for advanced OC $[60,61]$. Selected examples include dendritic cell vaccines, patient-specific autologous tumor cell vaccines, and vaccines targeting various antigens enriched in tumor cells, such as folate receptor alpha, HER2, brachyury, insulin-like growth factor binding protein-2, survivin, and carcinoembryonic antigen. These emerging therapies are of interest owing to the very limited treatment options for women who have failed 2 or more lines of chemotherapy, including platinum-based agents, and who have received or were ineligible to receive bevacizumab or a PARP inhibitor.

Another important aspect of addressing the unmet need for treatments in advanced $\mathrm{OC}$ is the regulatory pathway for accelerated approval in the United States [62, 63]. The Accelerated Approval Program allows earlier approval of drugs that treat serious conditions and fill an unmet need. In this program, oncology drugs can be approved based on a surrogate endpoint (such as objective response rate rather than survival), when those drugs fill an unmet need, have acceptable toxicity, and satisfy criteria for chemistry, manufacturing, and controls. Typically, approval is provisional and a confirmatory phase 3 trial is expected to be undertaken. This program has been beneficial for patients with $\mathrm{OC}$, which has lagged behind other cancers with regard to treatment options.

A good example of how accelerated approval has aided the OC community is PLD, which was granted accelerated approval in 1999. Under accelerated approval, which was based on three phase 2 studies, PLD was indicated for the treatment of metastatic $O C$ in patients with disease that was refractory to both paclitaxel- and platinum-based chemotherapy. According to the terms of the accelerated approval, a randomized, phase 3 clinical study was then completed to formally demonstrate the drug's clinical benefit in patients with relapsed OC $[64,65]$. On the basis of that trial, full approval of PLD was granted in 2005. More recently, the PARP inhibitor olaparib was granted accelerated approval in 2014 and the phase 3 SOLO-2 trial was submitted as a confirmatory study; the ongoing SOLO-3 trial may serve as a second confirmatory study. Rucaparib was also granted accelerated approval in 2016, with ARIEL3 and ARIEL4 serving as confirmatory studies. Given the continuing unmet need for therapies in many patients with $\mathrm{OC}$, it is encouraging that the accelerated approval program exists to usher in new treatment options in a manner that allows access that ensures an appropriate level of patient safety.

Despite new therapeutic strategies approved in recent years and promising strategies and agents on the horizon, there continue to be unmet needs for patients with advanced OC. Addressing those needs will require a reexamination and possibly a redesign of the drug discovery and development process. The cancer drug development process is facing many challenges, including inefficient clinical study designs, relative paucity of new drug targets but a proliferation of "me-too" drugs, and the dilution of the patient population available for enrollment into clinical studies. That dilution has many contributing causes, including the progress toward personalized medicine in which few patients may qualify for a given treatment, as well as the proliferation of clinical studies needed to test the large numbers of drugs with similar or identical mechanisms of action. Novel study designs including master protocols for umbrella, basket, and platform studies are being used to address this need [66]. Furthermore, many emerging therapies require biomarker tests, which must be developed and approved, and which are often expensive and provide low yields. These problems extend well beyond the OC arena, and their solutions call for a concerted and creative effort on the part of the scientific, pharmaceutical, and regulatory communities.

\section{Conclusions}

Platinum-based chemotherapy is recommended as first line therapy in women with advanced epithelial OC. Bevacizumab may have roles in selected patients with recurrent disease in combination with 5 approved chemotherapy backbones. For those patients who achieve an objective response to retreatment with platinum-based chemotherapy, a recent phase 3 study showed that niraparib can extend PFS when used as maintenance monotherapy. The recently reported phase 3 SOLO-2 study confirmed the efficacy of olaparib tablets as maintenance monotherapy in patients with platinum-sensitive, relapsed $\mathrm{OC}$ with a gBRCA mutation. Currently, there are limited treatment options for women with recurrent $\mathrm{OC}$ who have failed two or more lines of chemotherapy and have received or were ineligible to receive bevacizumab or a PARP inhibitor [39]. The US FDA's accelerated approval of olaparib and rucaparib for the treatment of recurrent disease offers exciting new treatment options. Accelerated FDA approval poses an opportunity for additional new medicines to become rapidly available to address unmet needs. 


\section{Abbreviations}

Cl: Confidence interval; CR: Complete response; DoR: Duration of response; gBRCA: germline BRCA; HR: Hazard ratio; HRD: Homologous recombination deficiency; ITT: Intent-to-treat; LOH: Loss of heterozygosity; NR: Not reported; OC: Ovarian cancer; ORR: Objective response rate; OS: Overall survival; PARP: Poly(ADP-ribose) polymerase; PFI: Platinum-free interval; PFS: Progression-free survival; PLD: Pegylated liposomal doxorubicin; QOL: Quality of life; SD: Standard deviation; VEGF: Vascular endothelial growth factor

\section{Acknowledgments}

The authors express thanks to Ken Scholz, PhD, Greg Tardie, PhD, Joan Hudson, and The Lockwood Group, Stamford, CT, for medical writing and editorial support, which was in accordance with Good Publication Practice (GPP3) guidelines.

\section{Funding}

Funding for medical writing and editorial support was provided by AstraZeneca LP.

\section{Availability of data and materials}

Not applicable.

\section{Authors' contributions}

Both authors were involved in drafting the article or revising it critically for important intellectual content, and both authors approved the final version to be submitted for publication. Development of the manuscript adhered to both authorship guidelines for Good Publication Practice and International Society for Medical Publication Professionals.

\section{Ethics approval and consent to participate}

Not applicable.

\section{Consent for publication}

Not applicable.

\section{Competing interests}

Dr. Monk discloses that he has received honoraria for speaker bureaus from Roche/Genentech, AstraZeneca, Janssen/Johnson \& Johnson, Myriad, Clovis, and TESARO. Additionally, Dr. Monk has been a consultant for Roche/ Genentech, Merck, TESARO, AstraZeneca, Gradalis, Advaxis, Cerulean, Amgen, Vermillion, ImmunoGen, Pfizer, Bayer, NuCana, Insys, GlaxoSmithKline, Verastem, Mateon (formally OxiGENE), Clovis, Precision Oncology, Perthera, Biodesix, and ImmunoGen. Dr. Herzog discloses that he has been a consultant for Roche, Johnson \&Johnson, AstraZeneca, TESARO, Clovis, and Caris within past 1 year.

\section{Publisher's Note}

Springer Nature remains neutral with regard to jurisdictional claims in published maps and institutional affiliations.

\section{Author details}

${ }^{1}$ University of Cincinnati Cancer Institute, University of Cincinnati, Medical Sciences Bldg, Suite 2005H, ML0662, 231 Albert Sabin Way, Cincinnati, OH 45267-0662, USA. ${ }^{2}$ Arizona Oncology (US Oncology Network), University of Arizona College of Medicine and Creighton University School of Medicine at Dignity Health St. Joseph's Hospital and Medical Center, Phoenix, AZ 85013, USA

Received: 13 July 2017 Accepted: 25 August 2017

Published online: 07 September 2017

\section{References}

1. Korkmaz T, Seber S, Basaran G. Review of the current role of targeted therapies as maintenance therapies in first and second line treatment of epithelial ovarian cancer; in the light of completed trials. Crit Rev Oncol Hematol. 2016;98:180-8.

2. Markman M. Maintenance chemotherapy in the management of epithelial ovarian cancer. Cancer Metastasis Rev. 2015;34(1):11-7.

3. Markman M, Liu PY, Wilczynski S, Monk B, Copeland LJ, Alvarez RD, et al. Phase III randomized trial of 12 versus 3 months of maintenance paclitaxel in patients with advanced ovarian cancer after complete response to platinum and paclitaxel-based chemotherapy: a southwest oncology group and gynecologic oncology group trial. J Clin Oncol. 2003;21(13):2460-5.

4. Markman M, Liu PY, Moon J, Monk BJ, Copeland L, Wilczynski S, et al. Impact on survival of 12 versus 3 monthly cycles of paclitaxel (175 mg/m2) administered to patients with advanced ovarian cancer who attained a complete response to primary platinum-paclitaxel: follow-up of a southwest oncology group and gynecologic oncology group phase 3 trial. Gynecol Oncol. 2009;114(2):195-8.

5. Pecorelli S, Favalli G, Gadducci A, Katsaros D, Panici PB, Carpi A, et al. Phase III trial of observation versus six courses of paclitaxel in patients with advanced epithelial ovarian cancer in complete response after six courses of paclitaxel/ platinum-based chemotherapy: final results of the After-6 protocol 1. J Clin Oncol. 2009;27(28):4642-8.

6. Copeland LJ, Brady MF, Burger RA, Rodgers WH, Huang H, Cella D, et al. A phase III trial of maintenance therapy in women with advanced ovarian/ fallopian tube/peritoneal cancer (O/PC/FT) after a complete clinical response (CCR) to first-line therapy: an NRG oncology study [late-breaking abstract]. National Harbor, Maryland: SGO Annual Meeting on Women's Cancer; 2017.

7. Burger RA, Brady MF, Bookman MA, Fleming GF, Monk BJ, Huang $H$, et al. Incorporation of bevacizumab in the primary treatment of ovarian cancer. $N$ Engl J Med. 2011;365(26):2473-83.

8. Perren TJ, Swart AM, Pfisterer J, Ledermann JA, Pujade-Lauraine E, Kristensen $\mathrm{G}$, et al. A phase 3 trial of bevacizumab in ovarian cancer. N Engl J Med. 2011;365(26):2484-96

9. Oza AM, Cook AD, Pfisterer J, Embleton A, Ledermann JA, Pujade-Lauraine E, et al. Standard chemotherapy with or without bevacizumab for women with newly diagnosed ovarian cancer (ICON7): overall survival results of a phase 3 randomised trial. Lancet Oncol. 2015;16(8):928-36.

10. Burger RA, Brady MF, Bookman MA, Monk BJ, Walker JL, Homesley HD, et al. Risk factors for $\mathrm{Gl}$ adverse events in a phase III randomized trial of bevacizumab in first-line therapy of advanced ovarian cancer: a gynecologic oncology group study. J Clin Oncol. 2014:32(12):1210-7.

11. Ray-Coquard IL, Harter P, Martin AG, Cropet C, Pignata S, Fujiwara K, et al. PAOLA-1: An ENGOT/GCIG phase III trial of olaparib versus placebo combined with bevacizumab as maintenance treatment in patients with advanced ovarian cancer following first-line platinum-based chemotherapy plus bevacizumab [abstract and poster presented at 2017 ASCO Annual Meeting]. J Clin Oncol. 2017;35(suppl):abstr TPS5605.

12. Pujade-Lauraine $E$, Combe P. Recurrent ovarian cancer. Ann Oncol. 2016; 27(Suppl 1):i63-5.

13. National Comprehensive Cancer Network. NCCN Clinical Practice Guidelines in Oncology. Ovarian Cancer Including Fallopian Tube Cancer and Primary Peritoneal Cancer. Version 1.2016. https://www.tri-kobe.org/nccn/guideline/ gynecological/english/ovarian.pdf. Accessed 15 March 2017.

14. Alberts DS, Liu PY, Wilczynski SP, Clouser MC, Lopez AM, Michelin DP, et al. Randomized trial of pegylated liposomal doxorubicin (PLD) plus carboplatin versus carboplatin in platinum-sensitive (PS) patients with recurrent epithelial ovarian or peritoneal carcinoma after failure of initial platinumbased chemotherapy (southwest oncology group protocol S0200). Gynecol Oncol. 2008;108(1):90-4.

15. Parmar MK, Ledermann JA, Colombo N, du Bois A, Delaloye JF, Kristensen $\mathrm{GB}$, et al. Paclitaxel plus platinum-based chemotherapy versus conventional platinum-based chemotherapy in women with relapsed ovarian cancer: the ICON4/AGO-OVAR-2.2 Trial. Lancet. 2003:361(9375):2099-106.

16. Pfisterer J, Plante M, Vergote I, du Bois A, Hirte H, Lacave AJ, et al. Gemcitabine plus carboplatin compared with carboplatin in patients with platinum-sensitive recurrent ovarian cancer: an intergroup trial of the AGO-OVAR, the NCIC CTG, and the EORTC GCG. J Clin Oncol. 2006:24(29):4699-707.

17. Raja FA, Counsell N, Colombo N, Pfisterer J, du Bois A, Parmar MK, et al. Platinum versus platinum-combination chemotherapy in platinum-sensitive recurrent ovarian cancer: a meta-analysis using individual patient data. Ann Oncol. 2013:24(12):3028-34.

18. Bruchim I, Jarchowsky-Dolberg O, Fishman A. Advanced (>second) line chemotherapy in the treatment of patients with recurrent epithelial ovarian cancer. Eur J Obstet Gynecol Reprod Biol. 2013;166(1):94-8.

19. Hanker LC, Loibl S, Burchardi N, Pfisterer J, Meier W, Pujade-Lauraine E, et al. The impact of second to sixth line therapy on survival of relapsed ovarian cancer after primary taxane/platinum-based therapy. Ann Oncol. 2012. 23(10):2605-12. 
20. Penson RT, Dignan F, Seiden MV, Lee H, Gallagher CJ, Matulonis UA, et al. Attitudes to chemotherapy in patients with ovarian cancer. Gynecol Oncol. 2004;94(2):427-35.

21. Cannistra SA, Matulonis UA, Penson RT, Hambleton J, Dupont J, Mackey H, et al. Phase II study of bevacizumab in patients with platinum-resistant ovarian cancer or peritoneal serous cancer. J Clin Oncol. 2007;25(33):5180-6.

22. Burger RA, Sill MW, Monk BJ, Greer BE, Sorosky Jl. Phase II trial of bevacizumab in persistent or recurrent epithelial ovarian cancer or primary peritoneal cancer: a gynecologic oncology group study. J Clin Oncol. 2007; 25(33):5165-71.

23. Monk BJ, Han E, Josephs-Cowan CA, Pugmire G, Burger RA. Salvage bevacizumab (rhuMAB VEGF)-based therapy after multiple prior cytotoxic regimens in advanced refractory epithelial ovarian cancer. Gynecol Oncol. 2006;102(2):140-4.

24. Pietzner K, Richter R, Chekerov R, Erol E, Oskay-Ozcelik G, Lichtenegger W, et al. Bevacizumab in heavily pre-treated and platinum resistant ovarian cancer: a retrospective study of the north-eastern German Society of Gynaecologic Oncology (NOGGO) ovarian cancer study group. Anticancer Res. 2011;31(8):2679-82.

25. Pujade-Lauraine E, Hilpert F, Weber B, Reuss A, Poveda A, Kristensen G, et al. Bevacizumab combined with chemotherapy for platinum-resistant recurrent ovarian cancer: the AURELIA open-label randomized phase III trial. J Clin Oncol. 2014;32(13):1302-8.

26. Aghajanian C, Blank SV, Goff BA, Judson PL, Teneriello MG, Husain A, et al. OCEANS: a randomized, double-blind, placebo-controlled phase III trial of chemotherapy with or without bevacizumab in patients with platinumsensitive recurrent epithelial ovarian, primary peritoneal, or fallopian tube cancer. J Clin Oncol. 2012;30(17):2039-45.

27. Aghajanian C, Goff B, Nycum LR, Wang YV, Husain A, Blank SV. Final overal survival and safety analysis of OCEANS, a phase 3 trial of chemotherapy with or without bevacizumab in patients with platinum-sensitive recurrent ovarian cancer. Gynecol Oncol. 2015;139(1):10-6.

28. Coleman RL, Brady MF, Herzog TJ, Sabbatini P, Armstrong DK, Walker JL, et al. Bevacizumab and paclitaxel-carboplatin chemotherapy and secondary cytoreduction in recurrent, platinum-sensitive ovarian cancer (NRG oncology/gynecologic oncology group study GOG-0213): a multicentre, open-label, randomised, phase 3 trial. Lancet Oncol. 2017;18(6):779-91.

29. Kaufman B, Shapira-Frommer R, Schmutzler RK, Audeh MW, Friedlander M, Balmana J, et al. Olaparib monotherapy in patients with advanced cancer and a germline BRCA1/2 mutation. J Clin Oncol. 2015;33(3):244-50.

30. Domchek SM, Aghajanian C, Shapira-Frommer R, Schmutzler RK, Audeh MW, Friedlander $\mathrm{M}$, et al. Efficacy and safety of olaparib monotherapy in germline BRCA1/2 mutation carriers with advanced ovarian cancer and three or more lines of prior therapy. Gynecol Oncol. 2016;140(2):199-203.

31. Gelmon KA, Tischkowitz M, Mackay H, Swenerton K, Robidoux A, Tonkin K, et al. Olaparib in patients with recurrent high-grade serous or poorly differentiated ovarian carcinoma or triple-negative breast cancer: a phase 2, multicentre, open-label, non-randomised study. Lancet Oncol. 2011;12(9): 852-61.

32. Swisher EM, Lin KK, Oza AM, Scott CL, Giordano H, Sun J, et al. Rucaparib in relapsed, platinum-sensitive high-grade ovarian carcinoma (ARIEL2 part 1): an international, multicentre, open-label, phase 2 trial. Lancet Oncol. 2017; 18(1):75-87.

33. Avastin (bevacizumab) [prescribing information]. South San Francisco: Genentech, Inc: 2016.

34. Clovis Oncology Inc. Rubraca (rucaparib) tablets [prescribing information]. Boulder, CO: Clovis Oncology, Inc.; 2016.

35. AstraZeneca Pharmaceuticals LP. Lynparza (olaparib) capsules [prescribing information]. AstraZeneca Pharmaceuticals LP: Wilmington, DE; 2017.

36. Tesaro Inc. Zejula (niraparib) capsules [prescribing information]. Waltham, MA: Tesaro, Inc.; 2017.

37. Ledermann J, Harter P, Gourley C, Friedlander M, Vergote I, Rustin G, et al. Olaparib maintenance therapy in platinum-sensitive relapsed ovarian cancer. N Engl J Med. 2012;366(15):1382-92.

38. Mirza MR, Monk BJ, Herrstedt J, Oza AM, Mahner S, Redondo A, et al. Niraparib maintenance therapy in platinum-sensitive, recurrent ovarian cancer. N Engl J Med. 2016;375(22):2154-64.

39. Pujade-Lauraine E, Ledermann JA, Penson RT, Oza AM, Korach J, Huzarski T, et al. Treatment with olaparib monotherapy in the maintenance setting significantly improves progression-free survival in patients with platinumsensitive relapsed ovarian cancer: results from the phase III SOLO-2 study (abstract and presentation slides). National Harbor, Maryland: SGO Annual Meeting on Women's Cancer; 2017.

40. Ledermann J, Harter P, Gourley C, Friedlander M, Vergote I, Rustin G, et al. Olaparib maintenance therapy in patients with platinum-sensitive relapsed serous ovarian cancer: a preplanned retrospective analysis of outcomes by BRCA status in a randomised phase 2 trial. Lancet Oncol. 2014;15(8):852-61.

41. Ledermann JA, Harter P, Gourley C, Friedlander M, Vergote I, Rustin G, et al. Overall survival in patients with platinum-sensitive recurrent serous ovarian cancer receiving olaparib maintenance monotherapy: an updated analysis from a randomised, placebo-controlled, double-blind, phase 2 trial. Lancet Oncol. 2016:17(11):1579-89.

42. Friedlander M, Gebski V, Gibbs E, Bloomfield R, Hilpert F, Wenzel LB, et al. Health-related quality of life (HRQOL) and patient-centered outcomes with maintenance olaparib compared with placebo following chemotherapy in patients with germline $(\mathrm{g})$ BRCA-mutated $(\mathrm{m})$ platinum-sensitive relapsed serous ovarian cancer (PSR SOC): SOLO-2 phase III trial [abstract presented at 2017 ASCO Annual Meeting]. J Clin Oncol. 2017;35(suppl):abstr 5507.

43. Mirza MR, Monk BJ, Gil-Martin M, Gilbert L, Canzler U, Follana P, et al. Efficacy of niraparib on progression-free survival (PFS) in patients (pts) with recurrent ovarian cancer (OC) with partial response (PR) to the last platinum-based chemotherapy [abstract and poster presented at 2017 ASCO Annual Meeting]. J Clin Oncol. 2017;35(suppl):abstr 5517.

44. Del Campo JM, Mirza MR, Berek JS, Provencher DM, Emons G, Fabbro M, et al. The successful phase 3 niraparib ENGOT-OV16/NOVA trial included a substantial number of patients with platinum resistant ovarian cancer (OC) [abstract and poster presented at 2017 ASCO Annual Meeting]. J Clin Oncol. 2017;35(suppl):abstr 5560.

45. Clovis Oncology Inc. Rucaparib significantly improved progression-free survival in all ovarian cancer patient populations studied in phase 3 ARIEL3 maintenance treatment trial (press release June 19, 2017). http://phx. corporate-ir.net/phoenix.zhtml?c=247187\&p=irol-newsArticle\&|D=2281511. Accessed 20 June 2017.

46. Clovis Oncology Inc. A study of rucaparib as switch maintenance following platinum-based chemotherapy in patients with platinum-sensitive, high-grade serous or endometrioid epithelial ovarian, primary peritoneal or fallopian tube cancer (ARIEL3). https://clinicaltrials.gov/ct2/show/NCT01968213?term= NCT01968213\&rank=1 Accessed: 20 June 2017.

47. Ramus SJ, Gayther SA. The contribution of BRCA1 and BRCA2 to ovarian cancer. Mol Oncol. 2009;3(2):138-50.

48. Hennessy BT, Timms KM, Carey MS, Gutin A, Meyer LA, Flake DD 2nd, et al. Somatic mutations in BRCA1 and BRCA2 could expand the number of patients that benefit from poly (ADP ribose) polymerase inhibitors in ovarian cancer. J Clin Oncol. 2010;28(22):3570-6.

49. Chetrit A, Hirsh-Yechezkel G, Ben-David Y, Lubin F, Friedman E, Sadetzki S. Effect of BRCA1/2 mutations on long-term survival of patients with invasive ovarian cancer: the national Israeli study of ovarian cancer. J Clin Oncol. 2008;26(1):20-5

50. Alsop K, Fereday S, Meldrum C. deFazio a, Emmanuel C, George J, et al. BRCA mutation frequency and patterns of treatment response in BRCA mutation-positive women with ovarian cancer: a report from the Australian ovarian cancer study group. J Clin Oncol. 2012;30(21):2654-63.

51. Pothuri B. BRCA1- and BRCA2-related mutations: therapeutic implications in ovarian cancer. Ann Oncol. 2013;24(Suppl 8):viii22-viii7.

52. AstraZeneca Pharmaceuticals LP. Olaparib treatment in relapsed germline breast cancer susceptibility gene (BRCA) mutated ovarian cancer patients who have progressed at least 6 months after last platinum treatment and have received at least 2 prior platinum treatments (SOLO-3). https:// clinicaltrials.gov/ct2/show/NCT02282020?term=NCT02282020\&rank=1 Accessed 12 May 2017.

53. Cadoo KA, Aghajanian C, Fraser C, Milner A, Kolvenbag G. A phase 2 study to assess olaparib by homologous recombination deficiency status in patients with platinum-sensitive, relapsed, ovarian, fallopian tube, or primary peritoneal cancer [abstract and poster presented at 2017 ASCO Annual Meeting]. J Clin Oncol. 2017;35(suppl):abstr TPS5606.

54. Liu JF, Barry WT, Birrer MJ, Lee JM, Buckanovich RJ, Fleming GF, et al. Overall survival and updated progression-free survival results from a randomized phase 2 trial comparing the combination of olaparib and cediranib against olaparib alone in recurrent platinum-sensitive ovarian cancer [abstract and poster presented at 2017 ASCO Annual Meeting]. J Clin Oncol. 2017; 35(suppl):abstr 5535 
55. AstraZeneca Pharmaceuticals LP. Olaparib or cediranib maleate and olaparib compared with standard platinum-based chemotherapy in treating patients with recurrent platinum-sensitive ovarian, fallopian tube, or primary peritoneal cancer. https://clinicaltrials.gov/ct2/show/NCT02446600?term= NCT02446600\&rank=1 Accessed 15 June 2017.

56. AstraZeneca Pharmaceuticals LP. Efficacy and safety study of cediranib in combination with olaparib in patients with recurrent platinum-resistant ovarian cancer (CONCERTO). https://linicaltrials.gov/ct2/show/ NCT02889900?term=NCT02889900\&rank=1 Accessed 15 June 2017.

57. National Cancer Institute. Cediranib maleate and olaparib or standard chemotherapy in treating patients with recurrent platinum-resistant or -refractory ovarian, fallopian tube, or primary peritoneal cancer. https:// clinicaltrials.gov/ct2/show/NCT02502266?term=NCT02502266\&rank=1 Accessed 15 June 2017.

58. Tesaro Inc. A study of niraparib in patients with ovarian cancer who have received three or four previous chemotherapy regimens (QUADRA). https:// clinicaltrials.gov/ct2/show/NCT02354586?term=NCT02354586\&rank=1 Accessed 12 May 2017

59. AstraZeneca Pharmaceuticals LP. AZD1775 plus chemotherapy in patients with platinum-resistant ovarian, fallopian tube, or primary peritoneal cancer. https://linicaltrials.gov/ct2/show/NCT02272790?term=NCT02272790\&rank=1 Accessed: 15 June 2017.

60. Leffers N, Daemen T, Boezen HM, Melief KJ, Nijman HW. Vaccine-based clinical trials in ovarian cancer. Expert Rev Vaccines. 2011;10(6):775-84.

61. Liao JB, Disis ML. Therapeutic vaccines for ovarian cancer. Gynecol Oncol. 2013;130(3):667-73.

62. United States Food and Drug Administration. Accelerated Approval Program 2016. https://www.fda.gov/drugs/resourcesforyou/healthprofessionals/ucm313768. htm Accessed: 12 May 2017.

63. United States Food and Drug Administration. Guidance for Industry: Expedited Programs for Serious Conditions - Drugs and Biologics. May 2014 Procedural. https:/wwwwdagov/downloads/drugs/guidancecomplianceregulatoryinformation/ guidances/ucm358301pdf Accessed: 12 May 2017

64. Gordon AN, Fleagle JT, Guthrie D, Parkin DE, Gore ME, Lacave AJ. Recurrent epithelial ovarian carcinoma: a randomized phase III study of pegylated liposomal doxorubicin versus topotecan. J Clin Oncol. 2001;19(14):3312-22.

65. Gordon AN, Tonda M, Sun S, Rackoff W. Doxil study investigators. Long-term survival advantage for women treated with pegylated liposomal doxorubicin compared with topotecan in a phase 3 randomized study of recurrent and refractory epithelial ovarian cancer. Gynecol Oncol. 2004;95(1):1-8.

66. Woodcock J, LaVange LM. Master protocols to study multiple therapies, multiple diseases, or both. N Engl J Med. 2017;377(1):62-70.

\section{Submit your next manuscript to BioMed Central and we will help you at every step:}

- We accept pre-submission inquiries

- Our selector tool helps you to find the most relevant journal

- We provide round the clock customer support

- Convenient online submission

- Thorough peer review

- Inclusion in PubMed and all major indexing services

- Maximum visibility for your research

Submit your manuscript at www.biomedcentral.com/submit

C) Biomed Central 